\title{
Fiabilidad de la autocalificación del alumnado de educación primaria. una experiencia durante las prácticas como maestra especialista de educación física
}

\section{Reliability of the self-calculation of the primary education pupil. An experience as teacher practices during physical education specialist}

\author{
Carla Fernández Garcimartín; Víctor M. López Pastor², y Cristina Pascual \\ Arias $^{3}$
}

Fecha de recepción: 16/01/2019; Fecha de revisión: 25/02/2019; Fecha de aceptación: 25/03/2019

Cómo citar este artículo:

Fernández-Garcimartín, C., López-Pastor, V. M., \& Pascual-Arias, C. (2018). Fiabilidad de la autocalificación del alumnado de educación primaria. Una experiencia durante las prácticas como maestra especialista de educación física. Revista de Innovación y Buenas Prácticas Docentes, 8, 97-109.

Autor de Correspondencia: Carla Fernández Garcimartín, carlafdzg96@gmail.com

Resumen:

En este trabajo mostramos una experiencia real del sistema de Evaluación Formativa y Compartida (EFyC) en un aula de primaria durante el periodo de prácticas. El objetivo de esta experiencia es analizar el rendimiento académico de los alumnos, así como la fiabilidad de la autocalificación del alumnado de quinto curso de primaria, comparando los resultados de las calificaciones de la maestra en prácticas con las del maestro tutor y las del propio alumnado. Para ello mostramos una breve fundamentación teórica sobre la EFyC que sirve de cimientos para nuestra experiencia, así como el diseño metodológico que hemos llevado a cabo. Los resultados de esta experiencia muestran una alta fiabilidad de este sistema de evaluación. En este sentido parece haber sido clave la participación del alumnado en su propia evaluación marcada con unos criterios establecidos y compartidos con el alumnado en todo momento.

Palabras clave: Autoevaluación, evaluación formativa, formación inicial, educación primaria.

Abstract:

In this paper we report a real experience of a Formative and Shared Evaluation in a primary school classroom during the internship period. The objective of this experience is to analyze. We have been able to analyze the student academic performance and reliability of the self-assessment by the fifth- grade, while comparing the results of the teacher education student in practice grades with those of the tutor and those of the students them-selves. For this, after a brief theoretical foundation on the formative and shared assessment that serves as the basis for our experience, we present the methodological design used and its implementation. The results of this experience show a high reliability of this evaluation system. In this sense, the participation of the students in their own assessment, marked with clear established criteria and shared with them at all times, seems to have been key.

Key Words: Self-assessment, Formative Assessment, Pre-Service Teacher Education, Primary Education.

\footnotetext{
${ }^{1}$ Universidad de Valladolid, Facultad de Educación de Segovia (España), carlafdzg96@gmail.com, CÓDIGO ORCID. 0000-0002-2171-0293

2 Universidad de Valladolid, Facultad de Educación de Segovia (España), vlopez@mpc.uva.es; CóDIGO ORCID. 0000-0003-2681-9543

3 Universidad de Valladolid, Facultad de Educación de Segovia (España), cristina.pascual@uva.es; CÓDIGO ORCID: 0000-0002-2781-5600
} 


\section{INTRODUCCIÓN}

La evaluación es uno de los elementos curriculares más importantes e influyentes en la educación y en el aprendizaje, dado que condiciona poderosamente los procesos de aprendizaje del alumnado (Berrocal, \& Berrocal, 2017; Herranz, \& López-Pastor, 2017). Los modelos de educación más tradicionales son meramente transmisivos y unidireccionales, y van ligados habitualmente a sistemas de evaluación sumativos y finales (López-Pastor, 2006; López, Monjas, \& Pérez, 2006; Herranz, \& López, 2017). Usando este modelo no se tienen en cuenta los aspectos del aprendizaje más difíciles de medir (aspectos subjetivos, individualizados, imposibilidad de cuantificarlos...) ya que salen fuera de esa objetivación que ofrece directamente la calificación. Tal como afirman López-Pastor, \& Pérez-Pueyo (2017), la mayoría de las personas que nos dedicamos a la educación es lo que hemos vivenciado durante nuestra vida como alumnos en el sistema escolar:

Las experiencias sobre la evaluación se limitan a aquellas que conocieron en su etapa previa a la universidad, y más recientemente durante su formación universitaria, en la que han primado los clásicos exámenes finales de carácter puramente sumativo, final y calificativo ( $p .387$ ).

De la crítica a los modelos tradicionales de evaluación se han dado nuevas propuestas que promueven un cambio de perspectiva en cuanto al proceso de evaluación y a sus consecuentes resultados en la educación. La evaluación "debe estar, siempre y en todos los casos, al servicio de quienes son los protagonistas en el proceso de enseñanza y de aprendizaje, y especialmente al servicio de los sujetos que aprenden" (Álvarez-Méndez, 2001, p. 3). Investigaciones como las de Santos-Guerra (2003), Hernández, \& Velázquez (2004), López-Pastor, González, \& Barba (2005), Fuentes, \& López (2017) y López Pastor, \& Pérez-Pueyo (2017) añaden que la implicación de los alumnos en la toma de decisiones de la evaluación es imprescindible, así como la aplicación de nuevas estrategias didácticas. De ahí nace el sistema de Evaluación Formativa y Compartida (EFyC), dentro de un marco que engloba un mayor compromiso por parte del docente en comparación con el sistema de evaluación tradicional.

En la figura 1 presentamos los conceptos y sus definiciones de este sistema de evaluación con el fin de comprender mejor la base de este sistema. Basada en LópezPastor, (2006), Herranz, \& López (2017), Blázquez (2017) y López-Pastor, \& PérezPueyo (2017):

\begin{tabular}{|c|c|c|}
\hline \multicolumn{3}{|c|}{ Conceptos de evaluación formativa y compartida } \\
\hline $\begin{array}{l}\text { Evaluación } \\
\text { formativa }\end{array}$ & $\begin{array}{l}\text { Proceso de evaluación que tiene como finalidad mejorar los } \\
\text { procesos de enseñanza y aprendizaje, favoreciendo así los } \\
\text { aprendizajes del alumno. Posibilita la reorientación de la enseñanza } \\
\text { durante el proceso con una finalidad meramente educativa. El } \\
\text { propósito de esta evaluación es disponer de suficiente información } \\
\text { del proceso de aprendizaje del alumno para que aprenda más junto } \\
\text { con el profesor. Se basa fundamentalmente en la observación del } \\
\text { alumno y el análisis de su trabajo. }\end{array}$ & $\begin{array}{l}\text { El estudiante: } \\
\text {-Toma } \\
\text { conciencia de su } \\
\text { progreso. } \\
\text {-Se } \\
\text { responsabiliza } \\
\text { de lo que hace. }\end{array}$ \\
\hline $\begin{array}{l}\text { Evaluación } \\
\text { compartida }\end{array}$ & $\begin{array}{l}\text { Proceso de diálogo en el que forma parte tanto el profesor como el } \\
\text { alumno. Tiene como finalidad que el alumno reflexione } \\
\text { individualmente sobre el proceso de aprendizaje, fomentando su } \\
\text { autonomía y su concienciación sobre lo que aprende. Es un proceso } \\
\text { de triangulación de la información (aprendizajes y procesos de } \\
\text { enseñanza-aprendizaje que se dan) para que no sea la opinión del } \\
\text { profesor la única que valga. }\end{array}$ & $\begin{array}{l}\text {-Aumenta la } \\
\text { motivación en lo } \\
\text { que aprende. }\end{array}$ \\
\hline
\end{tabular}

Figura 1. Conceptos de Evaluación Formativa y Compartida.

Fuente: Elaboración propia, basada en Blázquez, 2017; Herranz, \& López-Pastor, 2017; López-Pastor, 2006; y López-Pastor, \& Pérez-Pueyo, 2017). 
Desde nuestro punto de vista, este sistema de EFyC rompe con los sistemas de evaluación tradicionales cuya importancia recaía casi totalmente en la calificación. La evaluación debe ser un proceso de diálogo, una comprensión del trabajo realizado para considerar mejoras y ver la evolución del aprendizaje, así como un intercambio de conocimientos, sensaciones e ideas para aprender de manera más significativa (LópezPastor, 2007). El sistema de EFyC favorece tanto al maestro como al alumno en el ámbito educativo, desarrollando en el alumno aprendizajes de gran calidad cognitiva, motriz y actitudinal (López, Herranz, \& Mínguez, 2018). Como vemos en López-Pastor (2006), López-Pastor, González, \& Barba (2005), Herranz, \& López-Pastor (2017) y en López-Pastor, Herranz, \& Mínguez (2018) este sistema ofrece numerosos beneficios para ambos:

-Maestro: mayor implicación en la docencia, mejora de los procesos de enseñanza y aprendizaje (gracias a autoevaluaciones, observación sistemática, y demás instrumentos que veremos más adelante) y se da una enseñanza más individualizada consiguiendo así mayor conocimiento sobre los alumnos ayudando a que se formen integralmente.

-Alumno: favorece el análisis crítico y la autocrítica del trabajo personal, hay un mayor rendimiento académico, se da un valor formativo al aprendizaje ya que se supera la identificación de este con la calificación, mejora consigo el aprendizaje personal y favorece un intercambio de conocimientos y sensaciones junto con el maestro para tomarlo en consideración conjuntamente.

La EFyC en Educación Física está orientada en el aprendizaje del alumno, así como en su mejora. Para que el sistema de EFyC tenga un sentido práctico y funcional se requiere de unas técnicas e instrumentos de evaluación específicos (López-Pastor, \& Aldama, 2002; Herranz, \& López-Pastor, 2014; Blázquez, 2017 y López-Pastor, \& Pérez-Pueyo, 2017). En la figura 2 presentamos las técnicas e instrumentos más reconocidos por los autores indicados anteriormente:

\section{Técnicas e instrumentos de EFYC}

Sirve para anotar los aspectos más relevantes de las sesiones (cuando estas finalizan). Es un instrumento de gran validez para argumentar a los alumnos aspectos concretos de su aprendizaje, así como para analizar los puntos fuertes y débiles del trabajo del docente.

\begin{tabular}{|c|c|}
\hline $\begin{array}{l}\text { Cuaderno del } \\
\text { alumno }\end{array}$ & $\begin{array}{l}\text { Los alumnos anotan en él lo que se va a trabajar en la sesión, aspectos } \\
\text { claves de la unidad didáctica y las actividades que se llevan a cabo. Centran } \\
\text { al alumno en lo que se está trabajando (así siempre sabe dónde se } \\
\text { encuentra, se contextualiza lo que hace). }\end{array}$ \\
\hline $\begin{array}{l}\text { Fichas de } \\
\text { observación } \\
\text { grupales }\end{array}$ & $\begin{array}{l}\text { Muestran información de los alumnos para facilitar su proceso de } \\
\text { aprendizaje y para ayudarlos a llevar a cabo la evaluación y calificación. Es } \\
\text { una evaluación de lo observado en la sesión en relación a los objetivos } \\
\text { marcados. Si esta no se analiza, no se verá evolución en los alumnos. }\end{array}$ \\
\hline $\begin{array}{l}\text { Fichas de } \\
\text { seguimiento } \\
\text { individual }\end{array}$ & $\begin{array}{l}\text { Ayudan al maestro a tener recogida información sistemática de los procesos } \\
\text { de aprendizaje de cada alumno de forma individual, sistemática y continua. } \\
\text { Facilita una evaluación formativa más completa y real. }\end{array}$ \\
\hline $\begin{array}{l}\text { Cuestionarios } \\
\text { de } \\
\text { autoevaluación }\end{array}$ & $\begin{array}{l}\text { Cuestionarios en los que la evaluación es sobre uno mismo (tanto alumno } \\
\text { como maestro). Se puede realizar de forma individual o grupal. }\end{array}$ \\
\hline $\begin{array}{c}\text { Fichas de } \\
\text { tutocalificación }\end{array}$ & $\begin{array}{l}\text { El alumno se califica en relación al trabajo realizado según lo que se merece. } \\
\text { Aquí es cuando el profesor lo tiene en cuenta y llega a un acuerdo con el } \\
\text { alumno (negociación, intercambio de ideas). No se vería la calificación como } \\
\text { un hecho aislado del proceso de aprendizaje y de evaluación. }\end{array}$ \\
\hline
\end{tabular}

Figura 2. Instrumentos de EFyC.

Fuente: Elaboración propia basada en Blázquez, 2017; López-Pastor \& Aldama, 2002; Herranz \& López-

Pastor, 2014; y López-Pastor \& Pérez-Pueyo, 2017. 
Técnicas e instrumentos de EFYC

\begin{tabular}{|c|c|c|}
\hline \multirow{2}{*}{ 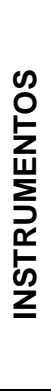 } & $\begin{array}{l}\text { Fichas de } \\
\text { coevaluación }\end{array}$ & $\begin{array}{l}\text { Evalúan entre iguales (alumno-alumno). Estas deben ser grupales y de } \\
\text { carácter colaborativo. }\end{array}$ \\
\hline & Escala graduada & $\begin{array}{l}\text { Instrumento que permite al maestro dar el salto entre el proceso de } \\
\text { evaluación formativa y continua y la calificación, bien al final del trimestre o } \\
\text { de cada UD. El alumno se posiciona constantemente con respecto a su nivel } \\
\text { de aprendizaje. En ella se destacan los objetivos o logros de aprendizaje a } \\
\text { alcanzar en relación con una calificación numérica y una valoración } \\
\text { cualitativa. }\end{array}$ \\
\hline \multirow{2}{*}{ 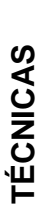 } & $\begin{array}{l}\text { Entrevistas } \\
\text { individuales y } \\
\text { grupales }\end{array}$ & $\begin{array}{l}\text { Se realizan entre el maestro y el o los alumnos para intercambiar } \\
\text { información, realizar correcciones y trabajar técnicas o tácticas de la sesión. } \\
\text { Se realizan al finalizar la unidad didáctica o al final de trimestre. }\end{array}$ \\
\hline & $\begin{array}{l}\text { Calific } \\
\text { dialo }\end{array}$ & $\begin{array}{l}\text { Es una consecuencia lógica de la evaluación compartida, ya que profesor y } \\
\text { alumno intercambian información y argumentos sobre la calificación para } \\
\text { llegar a un consenso. }\end{array}$ \\
\hline
\end{tabular}

Figura 3. Instrumentos de EFyC (continuación).

Fuente: Elaboración propia basada en Blázquez, 2017; López-Pastor \& Aldama, 2002; Herranz \& LópezPastor, 2014; y López-Pastor \& Pérez-Pueyo, 2017.

Estas técnicas e instrumentos se pueden llevar a cabo en todos los cursos de EP, aunque no obtendremos los mismos resultados con todos ellos. Gracias a relatos de experiencias prácticas como las que nos narran López-Pastor, González, \& Barba (2005), López-Pastor (2007, 2008) y López-Pastor, \& Pérez-Pueyo (2017), podemos afirmar que dependemos de varios factores para que la aplicación del sistema de EFyC en Educación Física sea óptima: (1) Madurez del alumnado: que los alumnos realicen una autoevaluación, autocalificación y/o coevaluación válida y objetiva, dependerá de su grado de madurez. Se ha comprobado que, cuanto más pequeños son los alumnos, menor veracidad hay en sus evaluaciones; (2) mostrar la evaluación de manera transparente: nada más comenzar las clases con los alumnos hay que ser claros y mostrarles cómo se va a evaluar, qué se va a utilizar para ello, cómo van a participar ellos y, sobre todo, qué ítems se van a tener en cuenta continuamente (en relación a los objetivos del maestro). Se concluye que, cuanta menos edad tengan los alumnos, más estructurados y claros deberán estar los instrumentos de evaluación que les proporcionemos (López-Pastor, 2006; López-Pastor, 2007).

\section{DESARROLLO DE LA EXPERIENCIA DE INNOVACIÓN}

Hemos organizado el desarrollo de la experiencia en diferentes apartados, de forma que pueda entenderse mejor la experiencia y el estudio llevados a cabo.

Objeto de estudio. El objeto de estudio de este trabajo es analizar la fiabilidad de la autocalificación del alumnado de quinto curso de primaria, así como comparar los resultados de las calificaciones de la maestra en prácticas con las del maestro tutor y las calificaciones dialogadas finales que se fijan con el alumnado en la entrevista final.

Diseño. El diseño de este estudio podría considerarse un estudio de caso, dado que se trata de una experiencia muy concreta en un solo grupo y con una muestra muy péquela. Ahora bien, en análisis utilizado es básicamente un análisis estadístico inferencial, concretamente un análisis de correlación.

Contexto. La experiencia se ha llevado a cabo en un centro educativo de la parte moderna de la ciudad de Segovia. Es un centro pequeño y familiar de una línea, que tiene solamente 210 alumnos. Las instalaciones del centro son muy completas y dispone de gimnasio cubierto, dos patios amplios y disponibilidad de uso de un pabellón polideportivo municipal muy cercano.

El grupo con el que se ha llevado a cabo la experiencia es el de $5^{\circ}$ de Primaria, que cuenta con 24 alumnos, o de los cuales son ACNEE. Aunque es un grupo muy 
heterogéneo, todos siguen un ritmo normal de clase, sin que eso afecte al aprendizaje. La experiencia se lleva a cabo en la asignatura de educación física (EF), que tiene asignadas dos horas y media semanales.

La maestra que lleva a cabo la experiencia lo realiza durante su periodo de prácticas como especialista de educación física. La experiencia de evaluación formativa y compartida (EFyC) se ha llevado a cabo durante una unidad didáctica (UD) con quinto curso de primaria. La UD "Me importa mi salud" está relacionada con el aprendizaje de los tipos de salud, la adquisición de hábitos saludables y la corrección de malos hábitos y mitos que se dan sobre la salud.

Sistema de calificación. El sistema de calificación utilizado consta de dos partes:

A. La maestra en prácticas lleva a cabo un sistema de calificación nuevo, ligeramente diferente al de su tutor. Este sistema está ajustado a los criterios de la figura 3 , diferenciados con una escala descriptiva. Desde el primer momento a los alumnos se les presenta esta figura 3 como el criterio más importante en el que nos vamos a apoyar para evaluarlos. Al finalizar la UD el alumnado tendrá que realizar un proceso de autoevaluación (Figura 3), según lo que ellos consideren de su evolución y aprendizaje en la UD (autoevaluándose). El profesor hará lo mismo sobre el trabajo de cada alumno basándose en los diferentes instrumentos de evaluación, dando una información coherente y objetiva del trabajo individual de cada alumno. Esta figura 3 se presenta a los alumnos con una escala verbal para no influenciar con la calificación numérica, eliminando poco a poco la importancia de la calificación sobre el aprendizaje.

\begin{tabular}{|c|c|c|c|c|}
\hline $\begin{array}{c}\text { Aspectos a } \\
\text { evaluar }\end{array}$ & Muy alto & Alto & Bajo & Muy bajo \\
\hline $\begin{array}{l}\text { Participar en } \\
\text { las sesiones } \\
\text { de manera } \\
\text { activa y } \\
\text { colaborativa. }\end{array}$ & $\begin{array}{l}\text { Siempre participo y me } \\
\text { ofrezco para realizar lo } \\
\text { que manden y ayudo a } \\
\text { mis compañeros sin } \\
\text { que me lo diga el } \\
\text { profesor. }\end{array}$ & $\begin{array}{l}\text { Normalmente } \\
\text { participo en las } \\
\text { sesiones y, a veces, } \\
\text { ayudo a mis } \\
\text { compañeros. }\end{array}$ & $\begin{array}{l}\text { Me cuesta participar } \\
\text { en las sesiones y } \\
\text { no suelo colaborar } \\
\text { con mis } \\
\text { compañeros. }\end{array}$ & $\begin{array}{l}\text { Apenas } \\
\text { participo en } \\
\text { las clases. } \\
\text { No colaboro } \\
\text { con mis } \\
\text { compañeros. }\end{array}$ \\
\hline $\begin{array}{l}\text { Reconocer } \\
\text { los errores } \\
\text { que } \\
\text { cometemos } \\
\text { en los tipos } \\
\text { de salud. }\end{array}$ & $\begin{array}{l}\text { Sé reconocer los } \\
\text { errores que cometemos } \\
\text { en todos los tipos de } \\
\text { salud y soy capaz de } \\
\text { corregirlos en mi día a } \\
\text { día. }\end{array}$ & $\begin{array}{l}\text { Sé reconocer los } \\
\text { errores que } \\
\text { cometemos en cada } \\
\text { tipo de salud que } \\
\text { trabajemos y estoy } \\
\text { intentando } \\
\text { corregirlos. }\end{array}$ & $\begin{array}{l}\text { Me cuesta } \\
\text { reconocer los } \\
\text { errores que se } \\
\text { cometen en cuanto } \\
\text { a la salud y no los } \\
\text { corrijo en mi día a } \\
\text { día. }\end{array}$ & $\begin{array}{l}\text { No } \\
\text { reconozco } \\
\text { los errores } \\
\text { que se } \\
\text { cometen en } \\
\text { cuanto a la } \\
\text { salud y no } \\
\text { los corrijo en } \\
\text { mi día a día. }\end{array}$ \\
\hline $\begin{array}{l}\text { Propongo } \\
\text { ideas } \\
\text { relacionadas } \\
\text { con lo que } \\
\text { estamos } \\
\text { trabajando } \\
\text { de la salud. }\end{array}$ & $\begin{array}{l}\text { Propongo ideas sobre } \\
\text { salud que aplico en mi } \\
\text { rutina. Se lo explico a } \\
\text { mis compañeros. }\end{array}$ & $\begin{array}{l}\text { Propongo algunas } \\
\text { ideas sobre el tipo de } \\
\text { salud a trabajar. Si } \\
\text { me lo piden mis } \\
\text { compañeros, se lo } \\
\text { explico. }\end{array}$ & $\begin{array}{l}\text { Me cuesta proponer } \\
\text { ideas sobre la salud } \\
\text { en nuestra vida si el } \\
\text { profesor no me pide } \\
\text { que lo haga. }\end{array}$ & $\begin{array}{l}\text { No propongo } \\
\text { ideas sobre } \\
\text { la salud en } \\
\text { nuestro día a } \\
\text { día aunque } \\
\text { me lo pida el } \\
\text { profesor. }\end{array}$ \\
\hline $\begin{array}{l}\text { Cumplo las } \\
\text { normas y } \\
\text { respeto al } \\
\text { material y a } \\
\text { mis } \\
\text { compañeros }\end{array}$ & $\begin{array}{l}\text { Siempre cumplo las } \\
\text { normas sin que el } \\
\text { profesor me llame la } \\
\text { atención. Corrijo a mis } \\
\text { compañeros cuando es } \\
\text { necesario. }\end{array}$ & $\begin{array}{l}\text { Cumplo las normas } \\
\text { sin que me lo diga el } \\
\text { profesor. }\end{array}$ & $\begin{array}{l}\text { Me cuesta cumplir } \\
\text { las normas y, por } \\
\text { eso, el profesor me } \\
\text { llama muchas } \\
\text { veces la atención. }\end{array}$ & $\begin{array}{l}\text { No cumplo } \\
\text { las normas. } \\
\text { Constantem } \\
\text { ente el } \\
\text { profesor me } \\
\text { llama la } \\
\text { atención. }\end{array}$ \\
\hline
\end{tabular}

Figura 4. Escala Descriptiva (o "Rúbrica") (continuación).

Fuente: Elaboración propia basada en López-Pastor, \& Pérez-Pueyo (2017). 


\begin{tabular}{|c|c|c|c|c|}
\hline $\begin{array}{c}\text { Aspectos a } \\
\text { evaluar }\end{array}$ & Muy alto & Alto & Bajo & Muy bajo \\
\hline $\begin{array}{l}\text { Realizo el } \\
\text { cuaderno } \\
\text { habitual- } \\
\text { mente, } \\
\text { anotando los } \\
\text { objetivos y } \\
\text { lo que } \\
\text { hacemos en } \\
\text { cada sesión }\end{array}$ & $\begin{array}{l}\text { Al terminar cada clase } \\
\text { hago el cuaderno como } \\
\text { me ha dicho el profesor } \\
\text { y de manera ordenada, } \\
\text { limpia y reflexiva. }\end{array}$ & $\begin{array}{l}\text { A veces hago el } \\
\text { cuaderno según } \\
\text { acaba la clase. A } \\
\text { veces sigo las pautas } \\
\text { que me ha mandado } \\
\text { el profesor: ordenado, } \\
\text { limpio y reflexivo. }\end{array}$ & $\begin{array}{l}\text { Me cuesta seguir el } \\
\text { ritmo de trabajo del } \\
\text { cuaderno. A veces } \\
\text { lo entrego tarde y/o } \\
\text { muy poco } \\
\text { elaborado. }\end{array}$ & $\begin{array}{l}\text { No entrego } \\
\text { el cuaderno } \\
\text { el día } \\
\text { marcado y/o } \\
\text { no me } \\
\text { esfuerzo en } \\
\text { hacerlo } \\
\text { correctament } \\
\text { e. }\end{array}$ \\
\hline $\begin{array}{l}\text { Escucho } \\
\text { activamente } \\
\text { las } \\
\text { explicacione } \\
\text { s del } \\
\text { profesor } \\
\end{array}$ & $\begin{array}{l}\text { Siempre escucho al } \\
\text { profesor sin que me } \\
\text { llame la atención. }\end{array}$ & $\begin{array}{l}\text { Escucho al profesor } \\
\text { aunque a veces me } \\
\text { llame la atención. }\end{array}$ & $\begin{array}{l}\text { No suelo escuchar } \\
\text { al profesor, me } \\
\text { llama la atención. }\end{array}$ & $\begin{array}{l}\text { No escucho } \\
\text { al profesor, } \\
\text { me llama la } \\
\text { atención } \\
\text { constanteme } \\
\text { nte. } \\
\end{array}$ \\
\hline $\begin{array}{l}\text { Comprendo } \\
\text { las } \\
\text { explicacione } \\
\text { s del } \\
\text { profesor. }\end{array}$ & $\begin{array}{l}\text { Comprendo lo que dice } \\
\text { porque se explica muy } \\
\text { bien y es agradable. }\end{array}$ & $\begin{array}{l}\text { Entiendo lo que dice } \\
\text { el profesor porque se } \\
\text { explica bien. }\end{array}$ & $\begin{array}{l}\text { A veces no } \\
\text { entiendo lo que dice } \\
\text { porque no se } \\
\text { explica muy bien. }\end{array}$ & $\begin{array}{l}\text { No entiendo } \\
\text { lo que dice } \\
\text { porque no se } \\
\text { explica bien. }\end{array}$ \\
\hline $\begin{array}{l}\text { El profesor } \\
\text { me ayuda a } \\
\text { aprender y a } \\
\text { mejorar. }\end{array}$ & $\begin{array}{l}\text { Siempre me ayuda en } \\
\text { lo que necesito, aún sin } \\
\text { pedirle ayuda. Me } \\
\text { explica las cosas sin } \\
\text { que yo le llame. }\end{array}$ & $\begin{array}{l}\text { Me ayuda siempre } \\
\text { que le pido ayuda y } \\
\text { me explica las cosas } \\
\text { si no las he } \\
\text { entendido. }\end{array}$ & $\begin{array}{l}\text { A veces me ayuda, } \\
\text { pero no siempre. Si } \\
\text { le llamo, me explica } \\
\text { las cosas. }\end{array}$ & $\begin{array}{l}\text { No me } \\
\text { ayuda nunca } \\
\text { ni me explica } \\
\text { las cosas } \\
\text { que no } \\
\text { entiendo. }\end{array}$ \\
\hline
\end{tabular}

Figura 5. Escala Descriptiva (o "Rúbrica") (continuación).

Fuente: Elaboración propia basada en López-Pastor, \& Pérez-Pueyo (2017).

Para finalizar con la parte de evaluación compartida se realizarán las entrevistas con cada alumno. Cada alumno cumplimentará una rúbrica como la establecida en la figura 3, al igual que el profesor, que la rellenará basándose en los instrumentos de evaluación completos según el proceso de aprendizaje de cada alumno. Tras el diálogo y el consenso (parte de la evaluación compartida), se pasa a convertir esos resultados verbales en numéricos, de forma que demos el salto a la "calificación dialogada". La nota final que se ponga es un número que abarca: aprendizajes individuales y grupales, mejoras a través de feedback, autoevaluaciones del trabajo propio, trabajo conceptual expuesto en el cuaderno, actitud y participación en clase. Al transformar la autoevaluación realizada de la figura 3 en autocalificación (tabla 1), se da un significado numérico al aprendizaje. En la tabla 1 se presentan los criterios de evaluación y la conversión de escala verbal a numérica para hacer más fácil el salto a la calificación.

Tabla 1.

Escala graduada para autoevaluación y autocalificación alumnado.

\begin{tabular}{lc}
\hline $\begin{array}{l}\text { Aspectos a valorar: Redondea con el bolígrafo las frases que mejor describan tu } \\
\text { trabajo y logros en esta unidad didáctica: }\end{array}$ & Grado \\
\hline -Siempre participo y me ofrezco para realizar lo que manden y ayudo a mis compañeros & MUY \\
sin que me lo diga el profesor. & ALTO \\
-Sé reconocer los errores que cometemos en todos los tipos de salud y soy capaz de & $(9-10)$ \\
corregirlos en mi día a día. & \\
-Propongo ideas sobre salud que aplico en mi rutina. Se lo explico a mis compañeros. & \\
-Siempre cumplo las normas sin que el profesor me llame la atención. Corrijo a mis & \\
compañeros cuando es necesario. & \\
-Al terminar cada clase hago el cuaderno como me ha dicho el profesor y de manera & \\
ordenada, limpia y reflexiva. & \\
\hline
\end{tabular}


Tabla 1.

Escala graduada para autoevaluación y autocalificación alumnado (continuación).

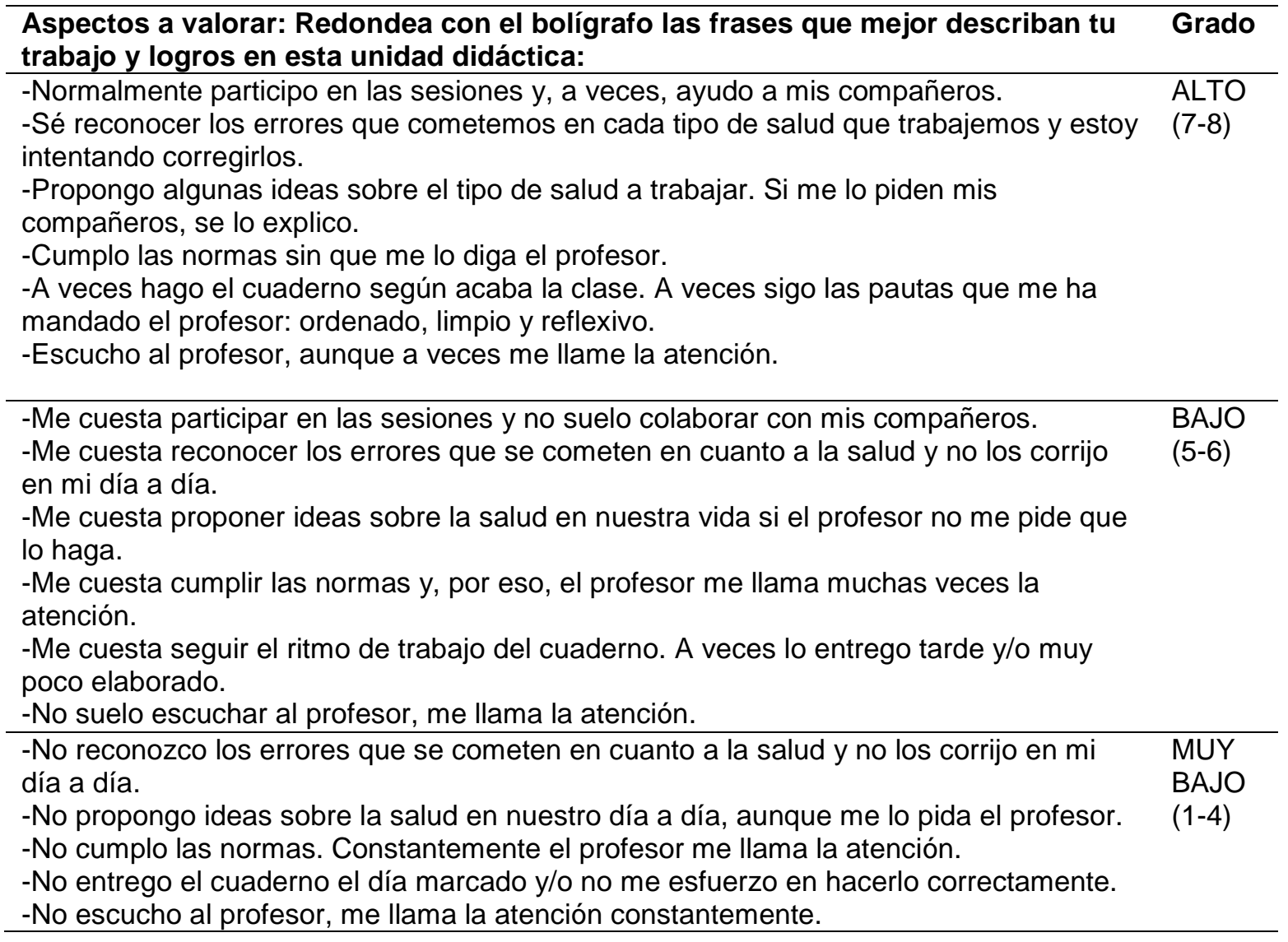

Una vez realizada la calificación de cada alumno, pasamos a un segundo paso:

B. Tras escribir la calificación con cada alumno, compararemos estos resultados con los que consigna su profesor-tutor. Esta comparación nos servirá para ver la fiabilidad de mi sistema de EFyC en comparación con el sistema de calificación global del tutor. El tutor del grupo sigue un sistema de calificación ligeramente diferente, basándose en:

(a) El cuaderno del alumno: los alumnos lo entregan semanalmente o cada dos semanas y el profesor lo corrige ese mismo día con feedback escrito dentro de él. Debe contener: qué se ha trabajo, qué han aprendido y un dibujo libre de la sesión. Si se entrega menos del $80 \%$ de las sesiones, se suspende este apartado y, por tanto, la evaluación de ese trimestre. En su cuaderno personal anota el registro de entregas del cuaderno.

(b) Trabajo diario: esfuerzo en las sesiones. A través de la observación sistemática en cada sesión, porque conoce a todos los alumnos y no necesita anotar datos en su cuaderno, únicamente el registro del cuaderno del alumno.

(c) Pruebas escritas: preguntas breves sobre el contenido trabajado. Los alumnos no necesitan estudiar. Casi nunca las lleva a cabo, pero lo tiene en su sistema por si lo usa.

(d) La actitud: ante el material, los compañeros, el profesor y las reglas. A través de la observación sistemática en cada sesión.

(f) La aptitud: cómo realiza los contenidos dados; por ejemplo: si les ha enseñado la técnica de carrera en relevos, si hace bien el pase de testigo y si sale trotando antes de que lo alcance su compañero.

A cada apartado pone una calificación numérica (4 al 10 y un guion "-" en caso de estar suspenso directamente en este apartado). Si el alumno tiene todos los 
apartados aprobados con más de un 4, hace una media aritmética para calcular la calificación final. Si no llega al 4 en alguno de estos puntos, suspende el trimestre.

\section{RESULTADOS}

Hemos organizado los resultados en dos grandes apartados: (a) el análisis del rendimiento académico del alumnado en esta unidad didáctica; $y$, (b) análisis de la fiabilidad de la autocalificación del alumnado de primaria y la correlación entre las calificaciones de la maestra en prácticas y el maestro tutor.

\subsection{Efecto en el rendimiento académico del alumnado}

En la tabla 2 mostramos los resultados académicos (en porcentajes y por número de alumnos) tras la puesta en práctica de la UD con $5^{\circ}$ de Primaria.

Tabla 2.

Resultados académicos de la UD realizada.

\begin{tabular}{ccc}
\hline & Resultados globales & \\
\hline Calificación & Porcentaje & $\mathbf{N}^{\mathbf{0}}$ alumnos/as \\
\hline Sobresaliente (9-10) & $20,83 \%$ & 5 \\
\hline Notable (7-8) & $62,5 \%$ & 15 \\
\hline Aprobado (5-6) & $16,66 \%$ & 4 \\
\hline Suspenso (1-4) & $0 \%$ & 0 \\
\hline Totales & $\mathbf{1 0 0} \%$ & $\mathbf{2 4}$ \\
\hline
\end{tabular}

Los resultados en el rendimiento académico del alumnado han sido muy positivos, tanto cuantitativamente (tabla 2) como cualitativamente. Cada alumno ha sido consciente del trabajo personal realizado durante la UD llevada a cabo, mostrándolo en cada autoevaluación, en su cuaderno y, concretamente, en la entrevista final.

Al pasar todo su esfuerzo y trabajo a una calificación numérica, fueron lo más justos posible, ya que la escala graduada que daba el salto a la calificación (tabla 1) no permitía salirse del baremo de notas donde se encontraba el alumno debido a su evaluación previa.

\subsubsection{Interpretación de dichos resultados.}

Como vemos en la tabla 2, ningún alumno ha suspendido la UD, debido a su implicación y trabajo continuo. Las calificaciones han sido muy altas y justas (habiendo coincidido con la mayoría de alumnos en la evaluación y posterior calificación), teniendo la mayoría de alumnos un notable (62.5\%) y más alumnos en el sobresaliente $(20.83 \%)$ que en el aprobado (16.6\%). Los alumnos con peores notas se encuentran en el aprobado, debido a su actitud (pasiva, desobediente) o bien al cuaderno (no lo entregaron al día, no hacían caso a las correcciones de mejora, etc.).

En el proceso de autocalificación, un alumno se puso como nota un 4, siendo consciente de que había faltado a tres de las seis sesiones de la UD y no había entregado el cuaderno. Tras la entrevista y la calificación dialogada, este alumno aprobó con un 5 debido a los criterios acordados en la escala descriptiva sobre cómo había trabajado esas tres sesiones.

\subsection{Resultados respecto a la fiabilidad de la autocalificación del alumnado y la correlación entre los dos maestros}

En la tabla 3 se presentan los datos, organizados en 5 columnas. Las calificaciones usan la escala habitual (0-10). 
Tabla 3.

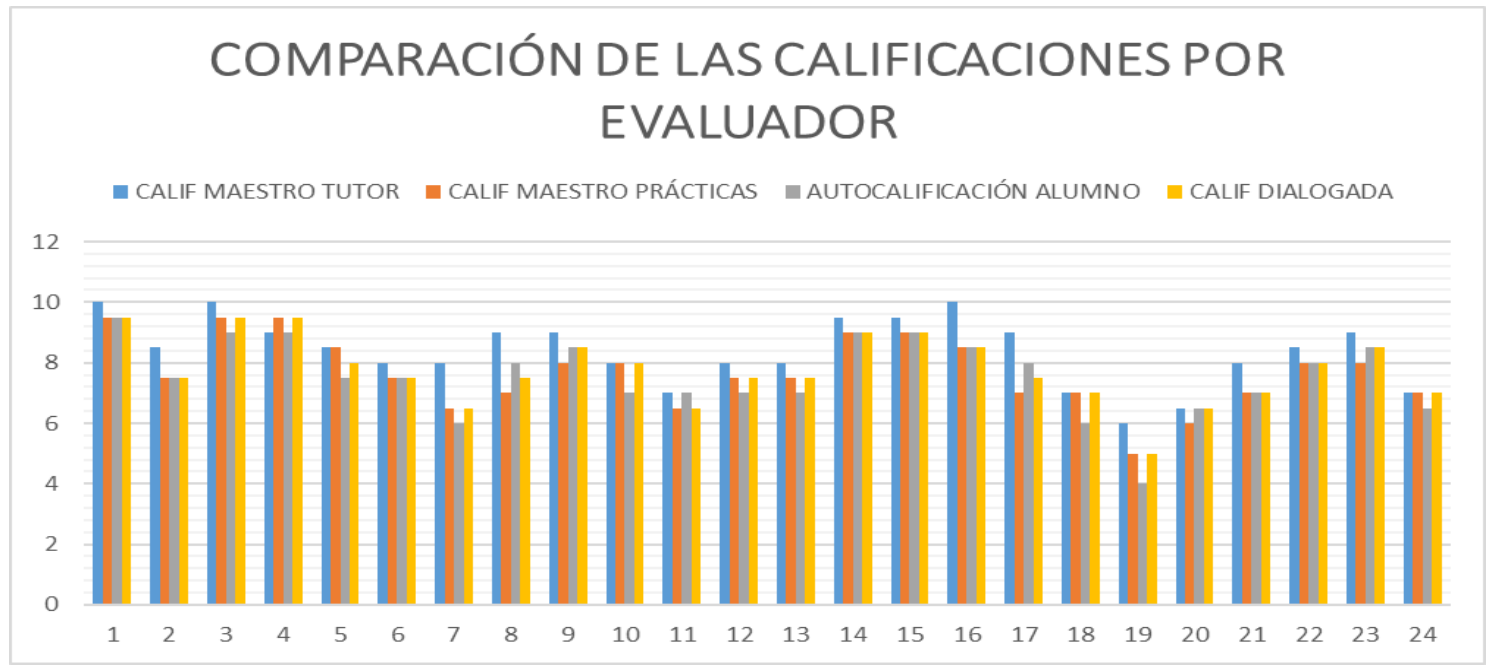

Calificaciones finales UD de $5^{\circ}$ Educación Primaria.

\begin{tabular}{|c|c|c|c|c|}
\hline \multicolumn{5}{|c|}{ Calificaciones finales } \\
\hline Alumno & $\begin{array}{c}\text { Calificaciones } \\
\text { del profesor } \\
\text { titular }\end{array}$ & $\begin{array}{l}\text { Calificaciones } \\
\text { del profesor } \\
\text { de prácticas }\end{array}$ & $\begin{array}{l}\text { Autocalificación del } \\
\text { alumnado }\end{array}$ & $\begin{array}{c}\text { Calificación } \\
\text { dialogada } \\
\text { (alumnado y } \\
\text { profesorado } \\
\text { de prácticas) }\end{array}$ \\
\hline 1 & 10 & 9,5 & 9,5 & 9,5 \\
\hline 2 & 8,5 & 7,5 & 7,5 & 7,5 \\
\hline 3 & 10 & 9,5 & 9 & 9,5 \\
\hline 4 & 9 & 9,5 & 9 & 9,5 \\
\hline 5 & 8,5 & 8,5 & 7,5 & 8 \\
\hline 6 & 8 & 7,5 & 7,5 & 7,5 \\
\hline 7 & 8 & 6,5 & 6 & 6,5 \\
\hline 8 & 9 & 7 & 8 & 7,5 \\
\hline 9 & 9 & 8 & 8,5 & 8,5 \\
\hline 10 & 8 & 8 & 7 & 8 \\
\hline 11 & 7 & 6,5 & 7 & 6,5 \\
\hline 12 & 8 & 7,5 & 7 & 7,5 \\
\hline 13 & 8 & 7,5 & 7 & 7,5 \\
\hline 14 & 9,5 & 9 & 9 & 9 \\
\hline 15 & 9,5 & 9 & 9 & 9 \\
\hline 16 & 10 & 8,5 & 8,5 & 8,5 \\
\hline 17 & 9 & 7 & 8 & 7.5 \\
\hline 18 & 7 & 7 & 6 & 7 \\
\hline 19 & 6 & 5 & 4 & 5 \\
\hline 20 & 6.5 & 6 & 6.5 & 6.5 \\
\hline 21 & 8 & 7 & 7 & 7 \\
\hline 22 & 8.5 & 8 & 8 & 8 \\
\hline 23 & 9 & 8 & 8.5 & 8.5 \\
\hline 24 & 7 & 7 & 6.5 & 7 \\
\hline
\end{tabular}

La figura 5 nos sirve para concluir de manera más visual la semejanza, dentro de cada alumno, que ha habido entre los dos sistemas de evaluación (EFyC y evaluación del maestro tutor). Está organiza en una gráfica en la que se incluyen 4 variables dentro del eje horizontal (cada alumno) y el eje vertical (notas 0-10): (1) Calificación del maestro tutor (azul); (2) calificación del maestro en prácticas (naranja); (3) autocalificación del alumno (gris) y (4) calificación dialogada (amarillo).

Lo más llamativo de la figura 5 es la ínfima diferencia que encontramos entre la calificación puesta por el maestro en prácticas y la autocalificación del alumno. Esto muestra un alto índice de credibilidad al sistema de EFyC. 
También se aprecian ciertas diferencias entre las calificaciones del maestro tutor y las del maestro en prácticas, siendo la primera algo más elevada (en la mayoría de los casos, menos en el alumno 4, 5, 10, 18 y 24, igualando la calificación del maestro en prácticas). Una posible explicación de estas diferencias es que el sistema de evaluación y de calificación usado por el maestro tutor no tiene en cuenta tantas variables como el sistema de EFyC que han utilizado tanto el maestro en prácticas y los alumnos. Por ello es normal que la calificación de los alumnos se aproxime más a la del maestro en prácticas, ya que han formado parte de su sistema de evaluación.

Las tres últimas variables arrojan valores semejantes en la mayoría de los casos. Desde el primer momento de la intervención se han mostrado los criterios, instrumentos y dinámica de evaluar tal y como eran, sin ocultarles a los alumnos variables que no pudieran tener en cuenta a la hora de conocer su evaluación. Este conocimiento inicial ha favorecido a que los resultados sean tan semejantes, ya que los mismos alumnos sabían sobre qué estaban siendo evaluados en todo momento. Los propios alumnos sabían que dependiendo de sus actuaciones iban a ser evaluados de una manera u otra y, además, conocían ese proceso de evaluación continua.

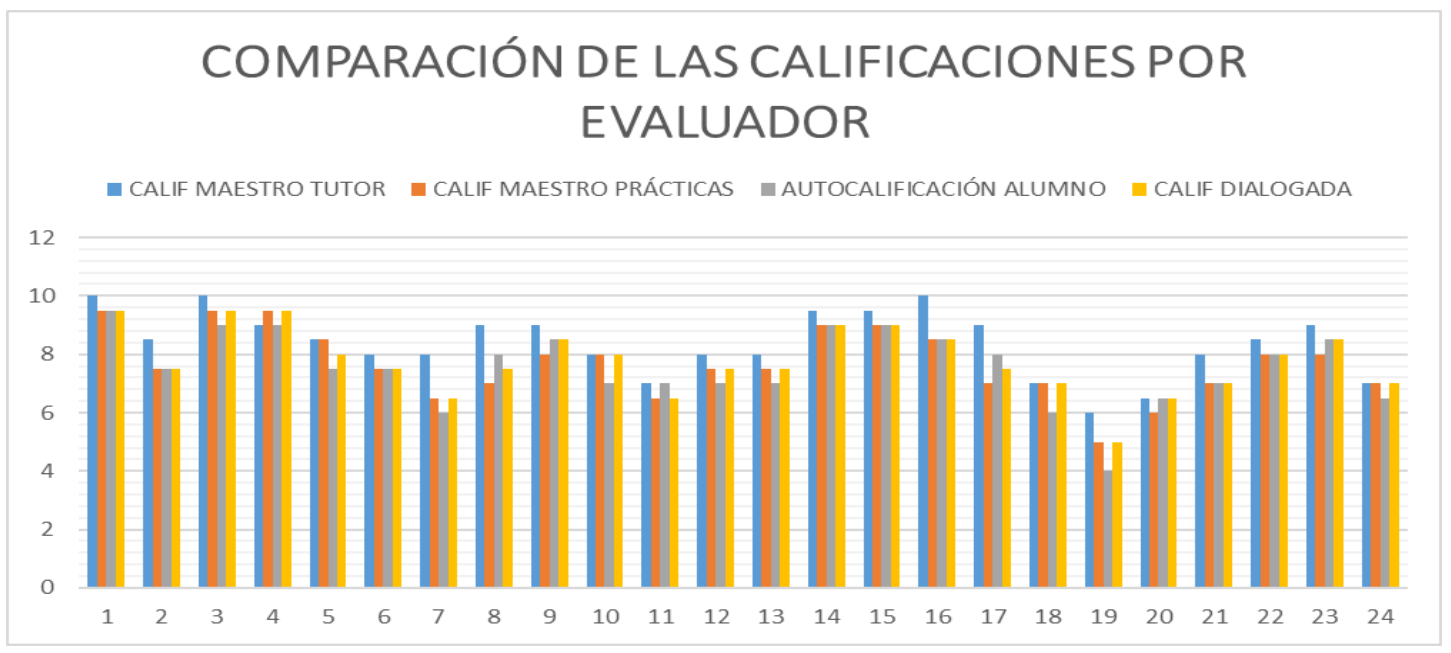

Figura 5. Gráfica comparativa de las calificaciones en función del evaluador.

Fuente: Elaboración propia.

Hemos utilizado como estadístico la correlación de Pearson, tomando las variables de 2 en 2, tal y como se muestra en la tabla 4. Además, presentamos los resultados y las conclusiones más significativas de las correlaciones entre las cuatro variables mencionadas en la tabla 3 y en la figura 5.

Tabla 4.

Correlación Pearson variables de evaluación.

\begin{tabular}{llcccc}
\hline & & \multicolumn{1}{c}{$\begin{array}{l}\text { Calificación } \\
\text { Maestro } \\
\text { Tutor }\end{array}$} & $\begin{array}{l}\text { Calificación } \\
\text { Maestro } \\
\text { Prácticas }\end{array}$ & $\begin{array}{l}\text { Autocalificació } \\
\text { n Alumno }\end{array}$ & $\begin{array}{l}\text { Calificación } \\
\text { Dialogada }\end{array}$ \\
\hline CalifMTutor & $\begin{array}{l}\text { Correlación de } \\
\text { Pearson }\end{array}$ & 1 & $.848^{\star *}$ & $.909^{\star \star}$ & .885 \\
& Sig. (bilateral) & & .000 & .000 & .000 \\
\cline { 2 - 6 } & $\mathrm{N}$ & 24 & 24 & 24 & 24 \\
\hline CalifMPrácticas & $\begin{array}{l}\text { Correlación de } \\
\text { Pearson }\end{array}$ & $.848^{\star \star}$ & 1 & $.883^{\star \star}$ & $.978^{\star \star}$ \\
\cline { 2 - 6 } & Sig. (bilateral) & .000 & & .000 & .000 \\
\cline { 2 - 6 } & $\mathrm{N}$ & 24 & 24 & 24 & 24 \\
\hline
\end{tabular}


Tabla 4.

Correlación Pearson variables de evaluación (continuación).

\begin{tabular}{llcccc}
\hline & & \multicolumn{1}{c}{$\begin{array}{l}\text { Calificación } \\
\text { Maestro } \\
\text { Tutor }\end{array}$} & $\begin{array}{l}\text { Calificación } \\
\text { Maestro } \\
\text { Prácticas }\end{array}$ & $\begin{array}{l}\text { Autocalificación } \\
\text { Alumno }\end{array}$ & $\begin{array}{c}\text { Calificación } \\
\text { Dialogada }\end{array}$ \\
\hline $\begin{array}{l}\text { Autocalificacion } \\
\text { Alumno }\end{array}$ & $\begin{array}{l}\text { Correlación de } \\
\text { Pearson }\end{array}$ & $.909^{\star \star}$ & $.883^{\star \star}$ & 1 & $.939^{\star \star}$ \\
\cline { 2 - 6 } & Sig. (bilateral) & .000 & .000 & 24 & .000 \\
\cline { 2 - 6 } & $\mathrm{N}$ & 24 & 24 & $.939^{\star \star}$ & 24 \\
\hline CalifDialogada & $\begin{array}{l}\text { Correlación de } \\
\text { Pearson }\end{array}$ & $.885^{\star \star}$ & $.978^{\star \star}$ & .000 & 1 \\
\cline { 2 - 6 } & Sig. (bilateral) & .000 & .000 & 24 & 24 \\
\cline { 2 - 6 } & $\mathrm{N}$ & 24 & 24 & & \\
\hline
\end{tabular}

**. La correlación es significativa en el nivel 0,01 (bilateral).

Los resultados indican que todas las correlaciones son muy altas (entre 0.848 y 0.978) y en todos los casos la significación es del 0.000 (absoluta). Por tanto, se trata de resultados estadísticamente significativos, y con un altísimo nivel de fiabilidad. Esto nos permite afirmar que existe una fuerte correlación entre las 4 variables estudiadas. Vamos a realizar un análisis más detallado entre las 6 posibles combinaciones dos a dos.

La correlación más alta (0.978) se da entre las calificaciones de la maestra de prácticas y la calificación dialogada (final), lo que nos podría indicar que, en los casos de duda, la calificación que predomina es la de la maestra.

La siguiente más alta $(0,939)$ es la que se da entre las auto-calificaciones del alumnado y la calificación dialogada (final), lo que nos indica al menos dos cosas: (a) la calificación del alumnado se ajusta mucho a lo que se merece; (b) la profesora tiene en cuenta los criterios y razonamientos del alumnado en el proceso de calificación dialogada.

Si analizamos la correlación entre la auto-calificación del alumnado y la maestra en prácticas (0.883) y el maestro tutor (0.909), podemos comprobar que la fiabilidad de la auto-calificación del alumnado es muy alta en los dos casos. Las diferencias respecto a los dos maestros son mínimas, aunque levemente superior respecto al maestro tutor.

Si analizamos la correlación entre la calificación de la maestra en prácticas y el maestro tutor (0.848), podemos comprobar que la fiabilidad es también muy alta, a pesar de ser la correlación más baja de las 6 . Podemos hacer diferentes interpretaciones de este dato: (a) los criterios e instrumentos de evaluación presentados por la maestra en prácticas se han adaptado perfectamente al contexto y a los alumnos (contexto que el maestro tutor conocía y por el que se basa, en gran parte, para evaluar a los alumnos); (b) la alta fiabilidad del trabajo de evaluación realizado por la maestra en prácticas demuestra que el trabajo previo a la evaluación ha sido muy riguroso, tanto que casi sus calificaciones finales son iguales que las del maestro tutor (el cual tiene mucha más experiencia y conocimientos que la maestra en prácticas). Esto nos da plena confianza para tomar en consideración la aplicación rutinaria de este sistema de EFyC.

Por otra parte, la correlación entre las calificaciones del maestro tutor y la calificación dialogada (final) es también muy alta (0.885), aunque la menor de las tres que hacen referencia a la calificación dialogada, lo que reforzaría las explicaciones anteriores de que podría ser debido a que: (a) la calificación final que ponga el maestro tutor se acerca mucho a la que realmente se merece el alumno (obtenida a través de la calificación dialogada); (b) ambos maestros tienen en cuenta unos criterios de evaluación reales que no se alejan de la realidad del alumno.

Habiendo analizado la experiencia realizada, comparto con Herranz \& LópezPastor (2014, p. 19) la siguiente idea:

La viabilidad de llevar a cabo procesos sistemáticos de autoevaluación, evaluación compartida, autocalificación y calificación dialogada en Educación Primaria, otorgando a los alumnos un papel activo y participativo, tanto en los procesos de evaluación como en 
los de calificación. Se trata de una alternativa educativamente valiosa frente al modelo tradicional de evaluación (p. 19).

\section{CONCLUSIONES}

Los resultados encontrados muestran que la autoevaluación y autocalificación del alumnado de $5^{\circ}$ de primaria que ha participado en esta experiencia es fiable. La experiencia se ha llevado a cabo en el área de Educación Física durante los tres meses de prácticas, incluyendo a todo el alumnado del grupo. En el apartado de resultados hemos mostrado cómo de fiable ha sido la autocalificación del alumnado basándonos en el análisis de la tabla 4 y de la figura 5. Entendemos que la alta fiabilidad alcanzada se debe, en parte, a tener unos criterios de evaluación claramente marcados que han sido compartidos con el alumnado en todo momento; también gracias al feedback continuo que se le ha dado a cada alumno, que permite ir mejorando de manera progresiva y ser continuamente reforzados de manera individual.

También se han hallado niveles de correlación muy altos y estadísticamente significativos entre las calificaciones de la maestra en prácticas, las del maestro tutor y las calificaciones dialogadas que se fijan con el alumnado en las entrevistas finales. Estos datos dotan de una elevada fiabilidad extra a todo el sistema desarrollado, dado que suponen una fuerte triangulación.

Las limitaciones encontradas son las siguiente: (a) al realizar la EFyC únicamente en una unidad didáctica y durante el periodo de prácticas la elaboración de los instrumentos de evaluación ha sido muy costoso y largo, mientras que si hubiéramos desarrollado el proceso de EFyC con los alumnos durante todo el curso podríamos haberlo rentabilizado mucho más; (b) el desconocimiento inicial de los alumnos sobre el sistema de EFyC, al que tuvieron que adaptarse rápidamente; (c) el desconocimiento de la maestra sobre dichos alumnos, al ser un grupo nuevo para ella al igual que una experiencia docente novedosa al tratarse de un periodo de prácticas;; (d) la muestra de alumnos puede considerarse limitada; (e) todo el estudio se ha realizado dentro del mismo contexto, por lo tanto no podemos generalizarlo a otros contextos o situaciones sin contar con las peculiaridades del mismo.

Consideramos que este estudio puede ser útil a todos los maestros y maestras que quieran iniciarse en el sistema de EFyC, así como a todos aquellos docentes que quieran seguir formándose dentro de este ámbito de evaluación. Puede ser especialmente relevante para alumnado de las carreras de magisterio que vayan a comenzar o estén realizando su periodo de prácticas docentes.

La principal prospectiva de futuro es seguir trabajando en este sistema de EFyC en las diferentes etapas del sistema educativo. De esta manera, enriqueceremos nuestras prácticas y vivencias, ampliaremos nuestra formación sobre la materia. Así seguiremos mejorando como docentes nuestro proceso de enseñanza y en consecuencia el aprendizaje de los alumnos.

\section{REFERENCIAS}

Álvarez-Méndez, J. M. (2001). Evaluar para conocer, examinar para excluir. Morata.

Berrocal, P. C., \& Berrocal, Ó. M. (2017). Recursos para las buenas prácticas y la evaluación formativa (Educación Infantil y Primaria). Revista Infancia, Educación y Aprendizaje, 3(2), 778-783.

Blázquez, D. (2017). Cómo evaluar bien Educación Física. El enfoque de la evaluación formativa. Barcelona: INDE.

Fuentes, T., \& López-Pastor, V. M. (2017). Evaluación auténtica, coevaluación y uso de las TIC en educación física: un estudio de caso en secundaria. Revista Infancia, Educación y Aprendizaje, 3(2), 42-46.

Hernández, J. L., \& Velázquez, R. (2004). La evaluación en educación física: Investigación y práctica en el ámbito escolar. Barcelona: Grao. 
Herranz, M., \& López-Pastor, V. M. (2014). ¿Es viable llevar a cabo procesos de autoevaluación y evaluación compartida en Educación Física en la etapa de Educación Primaria? un estudio de caso longitudinal (1 ${ }^{\text {a }}$ parte). Revista de educación física: Renovar la teoría y práctica, 133, 11-18.

Herranz, S., \& López-Pastor, V. M. (2017). Perspectiva del alumnado sobre su participación en los procesos de evaluación formativa y compartida en educación física en primaria: Un estudio de caso. EmásF: revista digital de educación física, 48, 27-48.

López, V. M., \& Aldama, B. (2002). Una experiencia de evaluación de actividades motrices en educación infantil. Aula de Innovación Educativa, 115, 19-23.

López-Pastor, V. M., \& Pérez-Pueyo, A. (coords.) (2017). Evaluación formativa y compartida en educación: experiencias de éxito en todas las etapas educativas. León: Universidad de León. (e-book). Recuperado de https://buleria.unileon.es/handle/10612/5999

López-Pastor, V. M. (2007). La evaluación en educación física y su relación con la atención a la diversidad del alumnado. Aportaciones, ventajas y posibilidades desde la evaluación formativa y compartida. Revista Kronos, 11, 59-71.

López-Pastor, V. M. (2008). Desarrollando sistemas de evaluación formativa y compartida en la docencia universitaria. Análisis de resultados de su puesta en práctica en la formación inicial del profesorado. European Journal of Teacher Education, 31(3), 293-311.

López-Pastor, V. M. (coord.) (2006). La evaluación en Educación Física. Revisión de modelos tradicionales y planteamiento de una alternativa: la evaluación formativa y compartida. Buenos Aires: Miñó y Dávila.

López-Pastor, V. M., \& Aldama, B. (2002). Una experiencia de evaluación de actividades motrices en educación infantil. Aula de Innovación Educativa, 115, 19-23.

López-Pastor, V. M., González, M., \& Barba, J. J. (2005). La participación del alumnado en la evaluación: la autoevaluación, la coevaluación y la evaluación compartida. Tándem, Didáctica de la Educación Física, 17, 21-37.

López-Pastor, V. M., Herranz, M., \& Mínguez, P. L. (2018). Evaluación formativa y compartida para una educación física crítica. En E. Lorente y D. Martos (Eds.), Educación Física y pedagogía crítica: Propuestas para la transformación personal y social (pp. 325-346). Universitat de Lleida y Universitat València.

López-Pastor, V. M., Monjas, R., \& Pérez-Brunicardi, D. (2003). Buscando alternativas a la forma de entender y practicar la Educación Física. Ed. INDE. Barcelona.

López-Pastor, V. M. \& Pérez-Pueyo, A. (coords.) (2017). Evaluación formativa y compartida en educación: experiencias de éxito en todas las etapas educativas. León: Universidad de León. (e-book). Recuperado de https://buleria.unileon.es/handle/10612/5999

Santos-Guerra, M. A. (2003). Una flecha en la diana: La evaluación como aprendizaje. Narcea Ediciones. Madrid. 